\begin{tabular}{|c|c|}
\hline Title & Effect of high-dimensional entanglement of Laguerre-Gaussian modes in parametric downconversion \\
\hline Author(s) & Kawase, Dai suke; Miyamoto, Y oko; Takeda, Mitsuo; Sasaki, Keiji; Takeuchi, Shigeki \\
\hline Citation & $\begin{array}{l}\text { Journal of the Optical Society of A merica B: Optical Physics, 26(4), 797-804 } \\
\text { https://doi.org/10.1364/JO SA B.26.000797 }\end{array}$ \\
\hline Issue Date & 2009-04-01 \\
\hline Doc URL & http:/hdl.handle.net/2115/49849 \\
\hline Rights & (c) 2009 Optical Society of A merica \\
\hline Type & article \\
\hline File Information & JOSA B26-4_797-804.pdf \\
\hline
\end{tabular}

Instructions for use 


\title{
Effect of high-dimensional entanglement of Laguerre-Gaussian modes in parametric downconversion
}

\author{
Daisuke Kawase, ${ }^{1}$ Yoko Miyamoto, ${ }^{2}$ Mitsuo Takeda, ${ }^{2}$ Keiji Sasaki, ${ }^{1}$ and Shigeki Takeuchi ${ }^{1,3, *}$ \\ ${ }^{1}$ Research Institute for Electronic Science, Hokkaido University, Sapporo 001-0020, Japan \\ ${ }^{2}$ Department of Information and Communication Engineering, University of Electro-Communications, \\ Chofu 182-8585, Japan \\ ${ }^{3}$ Institute of Scientific and Industrial Research, Osaka University, Ibaraki, Osaka 567-0047, Japan \\ *Corresponding author: takeuchi@es.hokudai.ac.jp
}

Received August 6, 2008; revised February 5, 2009; accepted February 25, 2009;

posted February 25, 2009 (Doc. ID 99844); published March 26, 2009

\begin{abstract}
We calculate the coincidence count probabilities when the photon pairs entangled in orbital angular momentum generated via spontaneous parametric downconversion are measured by using holograms with an $m$-fold dislocation, considering the nonzero crystal length. We find that the coincidence probabilities related to azimuthal changes in hologram positions deviate significantly from a sinusoidal curve, which has often been assumed in simple analyses. It is found that the main cause of this effect is the high-dimensional entanglement in Laguerre-Gaussian modes. We also find that the crystal length causes the hologram positions to change for the maximum coincidence probabilities. (C) 2009 Optical Society of America
\end{abstract}

OCIS codes: $050.4865,270.5585,270.5565$.

\section{INTRODUCTION}

The quantum states of photons in Laguerre-Gaussian (LG) modes have been attracting a great deal of attention recently. The LG modes form an orthogonal basis set of paraxial solutions to the wave equation and have screw phase dislocations $\exp (i l \phi)$, where $l$ is called the azimuthal mode index. In addition to a spin angular momentum (polarization), photons in LG modes carry an orbital angular momentum of $l \hbar[1]$. However, there is a significant difference between these two physical properties. In principle, an $N \times N$ Hilbert space with arbitrary large $N$ can be created from a single photon in the superposition of $N$ different LG modes [2-4], which is much larger than the $2 \times 2$ Hilbert space obtainable with polarization. Such quantum states, called quNits, can be used to improve quantum information protocols [5-7]. Furthermore, it is also interesting to study the nature of entanglement in larger Hilbert spaces by using single photons in LG modes. In this context, quantum entanglement in LG modes produced by spontaneous parametric downconversion (SPDC) has been studied experimentally [8-11]. Recently, states entangled both in polarization and in LG modes [12] and the control of entangled states in LG modes by using a geometrical phase (Gouy phase) [13] have been demonstrated.

In these experiments, holograms with a onefold dislocation have been used as LG mode converters. To detect a superposition of two different azimuthal modes, the optical axis of the incident light beam is shifted from the dislocation of the holograms. For the analysis of these experiments, the simple assumption that the measurement states are a superposition of only two azimuthal modes, i.e., $l=0$ and $l=+1$, has often been used. However, it has been pointed out that the measurement modes in such experiments must be considered a superposition of three or more azimuthal components of LG modes [14]. In this case, the effect of high-dimensional entanglement in LG modes of photon pairs [15-17] is not negligible.

In previous work, Padgett and co-workers reported a method for calculating the coincidence count probabilities of photon pairs generated by SPDC measured by using holograms with an $m$-fold dislocation [18,19]. Assuming that the crystal length is negligibly thin, they numerically calculated the coincidence rates when the hologram position is shifted from the center of the optical mode in the radial direction and found that the positions of zeros and the maximum coincidence probabilities strongly depend on the amount of the shift. However, the effect of highdimensional entanglement on the coincidence probabilities remained unclear, although Padgett and co-workers inferred that their result may be useful for quantitative analysis of entanglement.

In the present paper, we further explore the coincidence count probabilities of photon pairs generated by SPDC measured by using holograms with an $m$-fold dislocation. The differences from the previous analysis [19] are that the nonzero crystal length is considered and the coincidence probabilities are studied while changing the hologram positions two dimensionally (in the radial direction and azimuthal direction). Note that for a weakly focused pump beam, the crystal length, or the interaction length in the crystal, has a significant effect on the highdimensional entanglement of photons generated via SPDC [16,20]. Consequently, we have found that when the hologram positions are changing azimuthally the coincidence probabilities deviate from a sinusoidal curve 
[21-23], which has often been used in simple analyses that assume that photons are in the superposition of only two azimuthal modes. When the order of dislocation $m$ is larger, the deviations become more significant. Since the measurement modes contain a larger number of LG modes for larger $m$, we conclude that the main cause of the deviation is the high-dimensional entanglement in LG modes. We also find that the crystal length affects the hologram-position dependence of the coincidence count rates, resulting in the shift of the hologram positions for the maximum coincidence probabilities. This result is also important because the positions of zeros and the maximum coincidence probabilities may be used for quantitative analysis of entanglement [19].

The present paper is organized as follows. In Section 2, we explain the theory for the coincidence count probabilities of photon pairs generated by SPDC measured by using holograms with an $m$-fold dislocation, while taking the effect of nonzero crystal length into account. In Section 3 , we discuss in detail the calculated coincidence probabilities while changing the hologram positions two dimensionally. In Section 4, we present a summary and some concluding remarks.

\section{COINCIDENCE COUNT PROBABILITY}

A. Model of Experimental Equipment and Assumptions The experimental scheme for the measurement of quantum entanglement in spatial modes is shown in Fig. 1. The two-photon states are generated by SPDC in a nonlinear crystal and measured by two photon detectors while the positions of the two holograms $\left(\mathrm{H}_{s}\right.$ and $\left.\mathrm{H}_{I}\right)$ are changed; the holograms are used to convert measurement modes from a Gaussian mode in the single-mode fibers before the photodetectors to a superposition of LG modes.

Here, we assume that the pump beam used to generate photon pairs is a monochromatic continuous wave $(\mathrm{CW})$ and that the events where multiple pairs are created within the coherence time of the emitted photons are completely negligible. These assumptions are valid for typical experiments using a CW pump and a conventional bulk crystal, where a typical pair emission rate is $10^{6}$ pairs $/ \mathrm{s}$ and the coherence time of the emitted photons are about $10^{-12} \mathrm{~s}$ with a narrowband (nanometer) filter. The photon detectors create electronic signals when they detect single photons with a response rate that is sufficiently greater

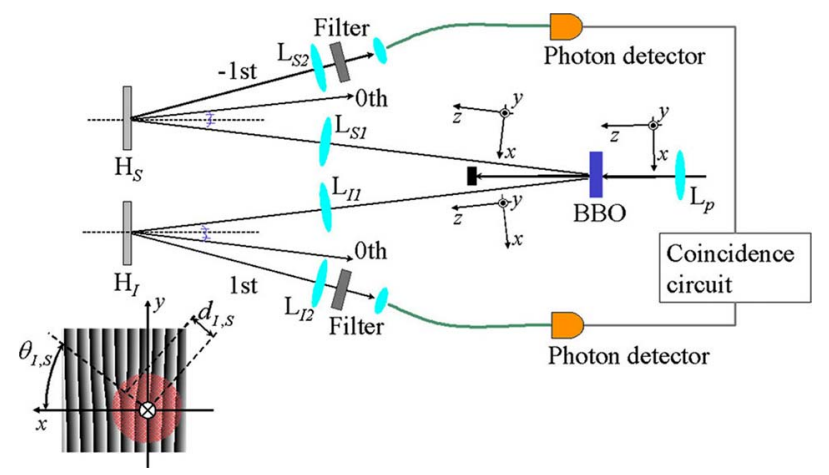

Fig. 1. (Color online) Experimental setup for measurement of quantum entanglement in spatial modes. The inset shows the scheme of the hologram $(m=1)$ as viewed from the BBO crystal. than the pair emission rate, and the coincidence circuit counts the events when the electronic signals are received from both of the photon detectors simultaneously. Since the multiple-pair emission is negligible, the photon detectors can be bucket detectors [24] or photon-number discriminating detectors $[25,26]$.

We also assume that the signal light and idler light are monochromatic, and we consider the situation in which the paraxial approximation with the propagation direction $z$ can be used. These conditions can be realized by using narrowband-pass filters (centered at $2 \lambda_{P}$, where $\lambda_{P}$ is the wavelength of the pump light) in the signal and idler paths, and small collection apertures. We also assume that the transverse size of the crystal is sufficiently larger than the waist size of the pump beam in the crystal. Under these conditions, we can assume that $\left|\mathbf{k}_{S, I, P}\right| \gg\left|\mathbf{q}_{S, I, P}\right|$, and $\left|\mathbf{k}_{S}\right| \approx\left|\mathbf{k}_{I}\right| \approx\left|\mathbf{k}_{P}\right| / 2$, where $\mathbf{k}_{S, I, P}$ is a wave vector and $\mathbf{q}_{S, I, P}$ is its transverse component for the $S$ (signal), $I$ (idler), and $P$ (pump) modes. In the proposed scheme, the pump light having a Gaussian profile is focused by a lens $\mathrm{L}_{P}$, and the beam waist is positioned in a nonlinear crystal with the beam radius of $w_{P}$. Here we assume that the pump is weakly focused; i.e., the Rayleigh range of the focused pump beam is larger than the crystal length $[13,27,28]$.

\section{B. Two-Photon State Generated by Parametric Downconversion}

The two-photon state in terms of the transverse component of the wave vector can be written as

$$
|\Psi\rangle_{\mathbf{q}}=\iint \mathrm{d} \mathbf{q}_{S} \mathrm{~d} \mathbf{q}_{I} \widetilde{\Phi}\left(\mathbf{q}_{S}, \mathbf{q}_{I}\right) a_{I}^{\dagger}\left(\mathbf{q}_{S}\right) a_{S}^{\dagger}\left(\mathbf{q}_{I}\right)|0,0\rangle,
$$

where $a_{S, I}^{\dagger}\left(\mathbf{q}_{S, I}\right)$ are creation operators for the idler and signal modes with the transverse component of wave vector $\mathbf{q}_{S, I},|0,0\rangle$ is the vacuum state, and the mode function $\widetilde{\Phi}\left(\mathbf{q}_{S}, \mathbf{q}_{I}\right)$ is written as follows [29,30]:

$$
\widetilde{\Phi}\left(\mathbf{q}_{S}, \mathbf{q}_{I}\right)=N_{\mathbf{q}} \exp \left(\frac{-w_{P}^{2}\left|\mathbf{q}_{S}+\mathbf{q}_{I}\right|^{2}}{4}\right) \operatorname{sinc}\left(\frac{L}{4 k_{P}}\left|\mathbf{q}_{S}-\mathbf{q}_{I}\right|^{2}\right),
$$

where $N_{\mathbf{q}}$ is normalization constant, $L$ is the crystal length, and $k_{P}$ is the magnitude of the wave vector of the pump light [31]. The sinc term of the mode function originates from the phase matching condition in the longitudinal direction. In some analyses using an approximation such that the crystal length $L$ is small, the sinc function is regarded as constant $[15,18,19]$. Here, we use a Gaussian approximation for the sinc function to simplify the integral calculus, and we rewrite the mode function of the two-photon state as follows [32-34]:

$$
\widetilde{\Phi}\left(\mathbf{q}_{S}, \mathbf{q}_{I}\right)=N_{\mathbf{q}}^{\prime} \exp \left(\frac{-w_{P}^{2}\left|\mathbf{q}_{S}+\mathbf{q}_{I}\right|^{2}}{4}\right) \exp \left(\frac{-b^{2}\left|\mathbf{q}_{S}-\mathbf{q}_{I}\right|^{2}}{4}\right),
$$

where $N_{\mathbf{q}}^{\prime}$ is a normalization constant, and we chose $b=1.42 \sqrt{L /\left(4 k_{P}\right)}$, which reproduces the width of the sinc function well [35]. 
The mode function in Fourier space $\widetilde{\Phi}\left(\mathbf{q}_{S}, \mathbf{q}_{I}\right)$ can be converted to that in position representation by inverse Fourier transformation:

$$
\begin{aligned}
\Phi\left(\mathbf{r}_{S}, \mathbf{r}_{I}\right) & =\iint \operatorname{d} \mathbf{q}_{S} \mathrm{~d} \mathbf{q}_{I} \widetilde{\Phi}\left(\mathbf{q}_{S}, \mathbf{q}_{I}\right) \exp \left(i \mathbf{q}_{S} \cdot \mathbf{r}_{S}\right) \exp \left(i \mathbf{q}_{I} \cdot \mathbf{r}_{I}\right) \\
& =N_{\mathbf{r}} \exp \left(-\left|\left(\mathbf{r}_{S}+\mathbf{r}_{I}\right) / 2\right|^{2} / w_{P}^{2}\right) \exp \left(-\left|\left(\mathbf{r}_{S}-\mathbf{r}_{I}\right) / 2\right|^{2} / b^{2}\right),
\end{aligned}
$$

where $\mathbf{r}_{S, I}$ represents the position in the transverse plane and $N_{\mathbf{r}}$ is a normalization constant.

Using Eq. (4), the two-photon state of Eq. (3) is written in position representation as follows:

$$
|\Psi\rangle_{\mathbf{r}}=\iint \mathrm{d} \mathbf{r}_{S} \mathrm{~d} \mathbf{r}_{I} \Phi\left(\mathbf{r}_{S}, \mathbf{r}_{I}\right) a_{I}^{\dagger}\left(\mathbf{r}_{S}\right) a_{S}^{\dagger}\left(\mathbf{r}_{I}\right)|0,0\rangle,
$$

where $a_{S, I}^{\dagger}\left(\mathbf{r}_{S, I}\right)$ are creation operators for the idler and signal modes at the transverse position $\mathbf{r}_{S, I}$, and $|0,0\rangle$ is the vacuum state.

\section{Measurement Modes}

We now turn to the calculation of the measurement modes in the idler and signal paths, which are determined by backpropagation. Since we use single-mode fibers before the photon detector, only the photons in Gaussian mode are counted. Thus, the Gaussian mode backpropagated through the lenses and holograms to the BBO crystal can basically be considered the measurement mode. For simplification of the notation, we define index $j$, which denotes the idler $(j=I)$ and signal $(j=S)$ paths. The backpropagated Gaussian mode from the single-mode fiber is focused by $\mathrm{L}_{j 2}$, and the beam waist is positioned at the plane of the hologram $\mathrm{H}_{j}$ with beam radius $w_{0}$. In our setup, the +1 st-order ( -1 st-order) diffraction beam is used in the idler (signal) path. When the position of $\mathrm{H}_{j}$ 's dislocations are $\left(d_{j}, \theta_{j}\right)$, as shown in Fig. 1 , the converted mode at the plane of the holograms is written as follows:

$$
\begin{aligned}
U^{m}\left(\rho_{j}, \phi_{j}\right)= & \exp \left[i \times \operatorname{sgn}(j) \times m \times \arg \left(\rho_{j} \cos \phi_{j}\right.\right. \\
& \left.\left.-d_{j} \cos \theta_{j}, \rho_{j} \sin \phi_{j}-d_{j} \sin \theta_{j}\right)\right] \sqrt{\frac{2}{\pi} \frac{1}{w_{0}}} \\
& \times \exp \left(-\rho_{j}^{2} / w_{0}^{2}\right),
\end{aligned}
$$

where $\left(\rho_{j}, \phi_{j}\right)$ denotes the cylindrical coordinates, $\operatorname{sgn}(j)$ represents $+1(-1)$ for $j=I(S)$, the function $\arg (x, y)$ gives the argument of the complex number $x+i y$, and $\left(\rho_{j} \cos \phi_{j}\right.$ $-d_{j} \cos \theta_{j}, \rho_{j} \sin \phi_{j}-d_{j} \sin \theta_{j}$ ) denotes the transverse position from the phase singularity in the plane of the holograms in rectangular coordinates.

The diffracted mode to which the singularity has been added is focused by $\mathrm{L}_{j 1}$, so that the beam waist is positioned at the plane of the $\mathrm{BBO}$ crystal. Here, we regard the backpropagated state at the BBO crystal as the measurement state. From Eq. (6), we obtain the measurement state $\left|\psi_{j}^{m}\left(d_{j}, \theta_{j}\right)\right\rangle$ at the plane of the BBO crystal, noting the inverted image $\left(\theta_{j} \rightarrow \theta_{j}+\pi\right)$, inverted by the action of the focusing lens:

$$
\begin{aligned}
\left|\psi_{j}^{m}\left(d_{j}, \theta_{j}\right)\right\rangle \propto & \int \exp \left[i \times \operatorname{sgn}(j) \times m \times \arg \left(\rho_{j} \cos \phi_{j}\right.\right. \\
& \left.\left.+d_{j} \cos \theta_{j}, \rho_{j} \sin \phi_{j}+d_{j} \sin \theta_{j}\right)\right] \sqrt{\frac{2}{\pi} \frac{1}{w_{0}}} \\
& \times \exp \left(-\rho_{j}^{2} / w_{0}^{2}\right) a_{j}^{\dagger}\left(\mathbf{r}_{j}\right)|0\rangle \mathrm{d} \mathbf{r}_{j},
\end{aligned}
$$

where $\left(\rho_{j} \cos \phi_{j}+d_{j} \cos \theta_{j}, \rho_{j} \sin \phi_{j}+d_{j} \sin \theta_{j}\right)$ denotes the transverse position from the phase singularity in the plane of the BBO crystal in rectangular coordinates.

\section{Coincidence Count Probability}

From Eqs. (5) and (7), the coincidence count probability as a function of $\mathrm{H}_{I}$ and $\mathrm{H}_{S}$ 's dislocation positions $\left(d_{I}, \theta_{I}, d_{S}, \theta_{S}\right)$ is given by

$$
\begin{aligned}
P_{m}\left(d_{I}, \theta_{I}, d_{S}, \theta_{S}\right)= & \mid\left\langle\psi_{I}^{m}\left(d_{I}, \theta_{I}\right)\left|\left\langle\psi_{S}^{m}\left(d_{S}, \theta_{S}\right) \mid \Psi\right\rangle_{\mathbf{r}}\right|^{2}\right. \\
\propto & \mid \iint \mathrm{d} \mathbf{r}_{S} \mathrm{~d} \mathbf{r}_{I} \Phi\left(\mathbf{r}_{S}, \mathbf{r}_{I}\right) \exp \left(-\rho_{S}^{2} / w_{0}^{2}\right) \\
& \times \exp \left(-\rho_{I}^{2} / w_{0}^{2}\right) \exp \{-i \times m \\
& \times\left[\operatorname { a r g } \left(\rho_{I} \cos \phi_{I}+d_{I} \cos \theta_{I}, \rho_{I} \sin \phi_{I}\right.\right. \\
& \left.\left.\left.+d_{I} \sin \theta_{I}\right)\right]\right\} \exp \left\{i \times m \times\left[\operatorname { a r g } \left(\rho_{S} \cos \phi_{S}\right.\right.\right. \\
& \left.\left.\left.+d_{S} \cos \theta_{S}, \rho_{S} \sin \phi_{S}+d_{I} \sin \theta_{I}\right)\right]\right\}\left.\right|^{2} .
\end{aligned}
$$

This coincidence probability includes the effects of all higher-order components of the spatial mode. In our scheme, the function of the coincidence probability $P_{m}\left(d_{I}, \theta_{I}, d_{S}, \theta_{S}\right)$ is characterized by only four parameters: the order of dislocation $m$, the beam radii of the pump light and measurement mode $\left(w_{P}\right.$ and $\left.w_{0}\right)$, and the crystal length $L$.

\section{E. Coincidence Probability in Terms of Laguerre-Gaussian Modes}

Next, to investigate which component of the LG modes contributes to the coincidence count probability $P_{m}$, we expand the photon states in Eq. (8), using LG modes.

A photon in the $\mathrm{LG}_{p l}$ mode carries an orbital angular momentum of $l \hbar$ [1]. The normalized LG mode at its beam waist is given in cylindrical coordinates by

$$
\begin{aligned}
\operatorname{LG}_{p l}(w ; \rho, \phi)= & \sqrt{\frac{2 p !}{\pi(|l|+p) !}} \frac{1}{w}\left(\frac{\sqrt{2} \rho}{w}\right)^{|l|} L_{p}^{|l|}\left(\frac{2 \rho^{2}}{w^{2}}\right) \\
& \times \exp \left(-\frac{\rho^{2}}{w^{2}}+i l \phi\right)
\end{aligned}
$$

where the other mode index $p$ is a label that is related to the number of radial nodes, the mode index $l$ is the order of the phase dislocation, $w$ is the beam radius at the beam waist, $(\rho, \phi)$ denote the cylindrical coordinates, and $L_{p}^{|l|}(\rho)$ are general Laguerre polynomials. 
Equation (8) can be expanded by using an orthogonal set of $\{|p l(w)\rangle\}$ by inserting $I=\Sigma_{p_{j}, l_{j}}\left|p_{j} l_{j}(w)\right\rangle\left\langle p_{j} l_{j}(w)\right|$, as follows:

$$
\begin{aligned}
P_{m}\left(d_{I}, \theta_{I}, d_{S}, \theta_{S}\right)= & \mid\left\langle\psi_{I}^{m}\left(d_{I}, \theta_{I}\right)\left|\left\langle\psi_{S}^{m}\left(d_{S}, \theta_{S}\right) \mid \Psi\right\rangle\right|^{2}\right. \\
= & \mid \sum_{l_{I}, l_{S}=-\infty}^{\infty} \sum_{p_{I}, p_{S}=0}^{\infty}\left\langle p_{I} l_{I}(w)\right| \\
& \times\left\langle p_{S} l_{S}(w) \mid \Psi\right\rangle_{\mathbf{r}}\left\langle p_{I} l_{I}(w) \mid \psi_{I}^{m}\left(d_{I}, \theta_{I}\right)\right\rangle^{*} \\
& \times\left.\left\langle p_{S} l_{S}(w) \mid \psi_{S}^{m}\left(d_{S}, \theta_{S}\right)\right\rangle^{*}\right|^{2}
\end{aligned}
$$

A photon state in the $\mathrm{LG}_{p l}$ mode can be written as [36]

$$
|p l(w)\rangle=\int \mathrm{d} \mathbf{r} \mathrm{LG}_{p l}(w ; \mathbf{r}) a^{\dagger}(\mathbf{r})|0\rangle
$$

where $\mathbf{r}=(\rho, \phi),|0\rangle$ is the vacuum state, and $a^{\dagger}(\mathbf{r})$ is the creation operator of a single photon at position $\mathbf{r}$.

Each component in Eq. (10) is written as follows. Since the mode function is assumed as Eq. (4), the amplitude of the two-photon state has a selection rule of azimuthal modes: $\left\langle p_{S} l_{S}(\omega)\right|\left\langle p_{I} l_{I}(\omega) \mid \Psi\right\rangle_{\mathbf{r}}=0$ for $l_{S}+l_{I} \neq 0$. Based on these results, the first component in Eq. (10) is written as follows:

$$
\left\langle p_{I} l_{I}(w)\right|\left\langle p_{S} l_{S}(w) \mid \Psi\right\rangle_{\mathbf{r}}=\delta_{-l_{S} l_{I}} C_{p_{S} l_{S}, p_{I} l_{I}}
$$

where $C_{p_{S} l_{S}, p_{I} l_{S}}$ is amplitude of a state $\left|p_{I} l_{I}(w)\right\rangle\left|p_{S} l_{S}(w)\right\rangle$.

Noting that $\arg \left(\rho_{j} \cos \phi_{j}+d_{j} \cos \theta_{j}, \rho_{j} \sin \phi_{j}+d_{j} \sin \theta_{j}\right)$ $=\theta_{j}+\arg \left[\rho_{j} \cos \left(\phi_{j}-\theta_{j}\right)+d_{j}, \rho_{j} \sin \left(\phi_{j}-\theta_{j}\right)\right]$ and that $\mathrm{LG}_{p l}(w ; \rho, \phi)=\exp (i l \theta) \mathrm{LG}_{p l}(w ; \rho, \phi-\theta)$, we obtain

$$
\begin{aligned}
\left\langle p_{j} l_{j}(w) \mid \psi_{j}^{m}\left(d_{j}, \theta_{j}\right)\right\rangle= & \left.\exp \left\{i \times \operatorname{sgn}(j) \times m \times \theta_{j}\right)\right\} \\
& \times \exp \left(-i l_{j} \theta_{j}\right) \alpha_{p_{j} l_{j}}^{m, j}\left(d_{j}\right),
\end{aligned}
$$

where $\alpha_{p_{j}, l_{j}}^{m, j}\left(d_{j}\right)=\left\langle p_{j} l_{j}(w) \mid \psi_{j}^{m}\left(d_{j}, 0\right)\right\rangle$.

From Eqs. (12) and (13), the coincidence count probability, Eq. (10), can be rewritten as follows:

$$
P_{m}=\left|\sum_{l=-\infty}^{\infty} \exp \left\{i l\left(\theta_{I}-\theta_{S}\right)\right\} R_{l}^{m}\left(d_{S}, d_{I}\right)\right|^{2},
$$

where $R_{l}^{m}$ is written as follows:

$$
R_{l}^{m}\left(d_{S}, d_{I}\right)=\sum_{p_{S}, p_{I}=0}^{\infty} \alpha_{p_{S^{-l}}}^{m, S^{*}}\left(d_{S}\right) \alpha_{p_{I}}^{m, I^{*}}\left(d_{I}\right) C_{p_{S^{-}-p_{I}}}
$$

Note that $R_{l}^{m}$ does not depend on the chosen set $\{|p l(w)\rangle\}$ with any $w$.

Equation (14) shows that $P_{m}$ is a function of only the difference of azimuthal positions $\left(\theta_{I}-\theta_{S}\right)$ when radial positions $\left(r_{S}, r_{I}\right)$ are fixed. This conclusion is natural when we consider the symmetry of the LG modes in the azimuthal direction.

\section{SIMULATION RESULTS AND DISCUSSION}

\section{A. Two-Dimensional Maps of Coincidence Count Probability}

Using the experimental setup shown in Fig. 1, 2D maps of coincidence count probabilities can be measured by scanning the position of $\mathrm{H}_{S}$ two dimensionally (the azimuthal position $\theta_{S}$ and the radial position $d_{S}$ ) for a given position of $\mathrm{H}_{I}$. Note that during the entire scan the hologram remains within a single plane and the orientation of the hologram pattern is kept constant.

Figure 2 shows the 2D maps of coincidence count probabilities, which we numerically calculated by using Eq. (8). Since the probability depends only on the difference of azimuthal positions $\left(\theta_{I}-\theta_{S}\right)$ when radial positions $\left(d_{S}, d_{I}\right)$ are fixed (see Subsection 2.E), the 2D maps are calculated for a fixed $\theta_{I}(=0)$. The amount of hologram shift is normalized by the beam radius of the backpropagated mode $\left(w_{0}\right)$, which is now assumed to be sufficiently smaller than the transverse size of the crystal. For the following analysis, we chose the parameters to be $w_{P} / w_{0}=2$ and $b / w_{0}$ $=0.102$. In an actual experimental setup, these parameters correspond to $w_{P}=170 \mu \mathrm{m}, w_{0}=85 \mu \mathrm{m}$, and $L$ $=3 \mathrm{~mm}$ with $\lambda_{P}=351 \mathrm{~nm}[13,37]$.

First, we consider the case of $m=1$. When $d_{I}=0$ [Fig. 1(a)], the peak exists in the origin. The reason is as follows. The measurement mode in the idler path consists of only the azmithal component $l_{I}=1$, because the dislocation of the hologram exists in the optical axis $\left(d_{I}=0\right)$. Owing to the nature of the quantum entanglement explained in Eq. (12), the projected mode in the signal path consists of only the azimuthal mode $l_{S}=-1$. In this case, the conversion efficiency from the projected mode to the detection mode $\left(\mathrm{LG}_{00}\right)$ by the hologram is maximum when the dislocation of the hologram is set in the optical axis $\left(d_{S}=0\right)$. This is the reason that the peak appears at the origin when $d_{I}=0$.

When $\left(d_{I}, \theta_{I}\right)=(0.5,0),(1.0,0),(1.5,0)$ [Figs. 1(b)-1(d)], the positions of hologram dislocation giving the maximum coincidence count probability are $\left(d_{S}, \theta_{S}\right)$ $=(0.5,0),(1.1,0),(1.6,0)$, respectively. These results suggest that the coincidence count probability is maximum when $d_{S}$ is almost $d_{I}$. This phenomenon is explained intuitively as follows. Similar to the case of $d_{I}=0$, the singularity position $\left(d_{I}, 0\right)$ of the measurement mode in the idler path is effectively projected onto the idler path because of the entanglement. The conversion efficiency of the projected mode to $\mathrm{LG}_{00}$ is maximum when the hologram dislocation is positioned at the singularity of the projected mode. Thus, the coincidence becomes maximum when $d_{S}$ is almost $d_{I}$. A detailed discussion of this issue will be presented in Subsection 3.C.

The coincidence probabilities for the cases of $m=2,3$ are shown in Figs. 2(e)-2(l). It is interesting to see that for all of these cases, the coincidence count probability is maximum when $d_{S}$ is almost $d_{I}$. This phenomenon is common for the case of $m=1$. However, the distribution of coincidence probabilities changes significantly as $m$ increases. That is, the areas of low coincidence probabilities, indicated by the blue regions, spread more widely when $m$ increases. For example, when the disloca- 

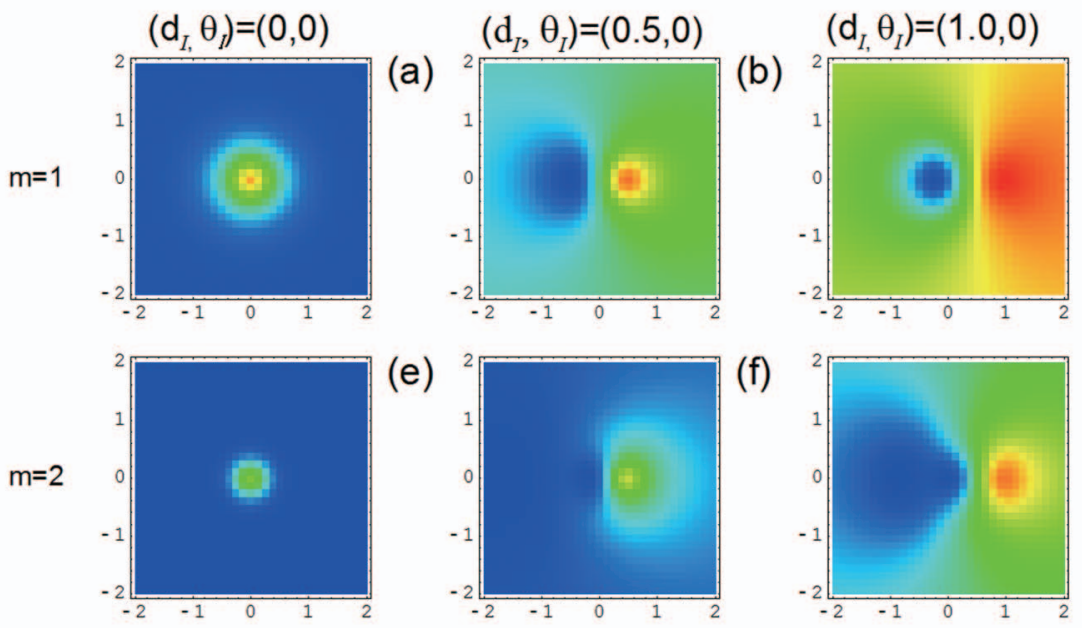

(e)
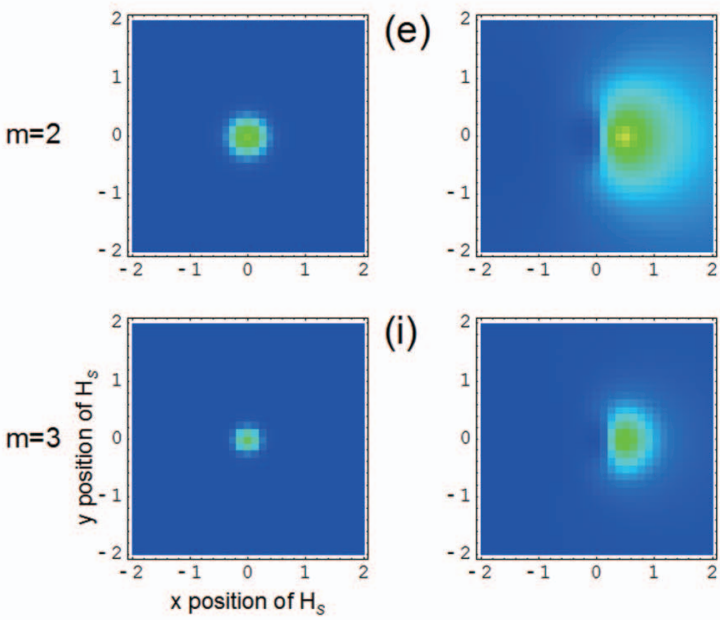

(i)

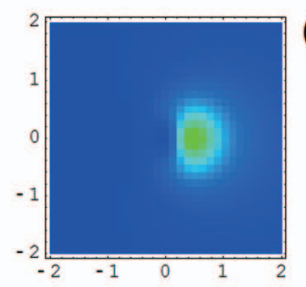

(f)

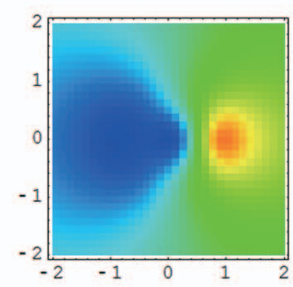

(j)

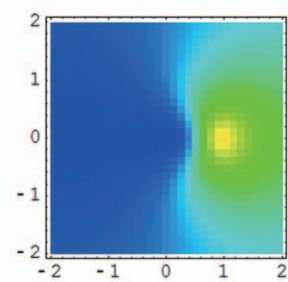

(c)

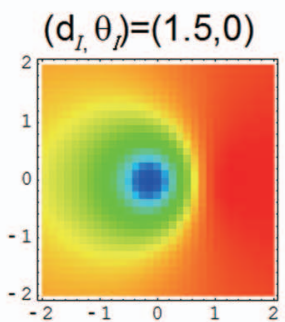

(d)

(g)

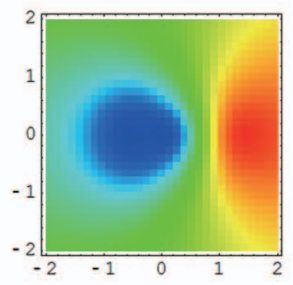

(h)

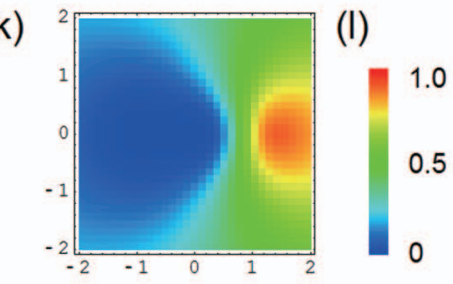

Fig. 2. 2D maps of the coincidence count probabilities $P_{m}\left(d_{I}, \theta_{I}, d_{S}, \theta_{S}\right)$ when the position of $\mathrm{H}_{S}$ is scanned for four different positions of $\mathrm{H}_{I}$ and for three different dislocation orders. The columns show maps for $\left(d_{I} / w_{0}, \theta_{I}\right)=(0,0),(0.5,0),(1.0,0),(1.5,0)$, and the rows show maps for $m=1,2,3$. Parameters are $w_{P} / w_{0}=2$ and $b / w_{0}=0.102$. The coincidence probabilities are normalized by the coincidence probability for $m=0$ in each $2 \mathrm{D}$ map.

tion position is $\left(d_{I}, \theta_{I}\right)=(1.0,0)$, the low-coincidenceprobability area is localized around $\left(d_{S}, \theta_{S}\right)=(0.2, \pi)$ for $m=1$ [Fig. 2(c)]. However, the low-coincidence-probability area spreads when $m=2$ [Fig. $2(\mathrm{~g})$ ], and for $m=3$ [Fig. $2(\mathrm{k})]$ most of the region with $x_{S} \equiv d_{S} \cos \theta_{S}<0$ is occupied by the low-coincidence-probability region. In the next subsection, we will discuss how these changes influence the experiments in which the phases of the measurement states are controlled by scanning the hologram azimuthally.

\section{B. One-Dimensional Scan in Azimuthal Direction}

To change the relative phases between the measurement basis, the method of scanning the azimuthal position of the hologram dislocation $\theta_{S, I}$ while fixing $d_{S, I}$ has been used in a number of experiments [8-14,38]. In the experiments in which holograms with a onefold dislocation are used, the measurement modes have often been assumed to have only two basis states. Under this simple assumption, the coincidence probability as a function of $\theta$ becomes a sinusoidal curve, shown as a solid curve in Fig. 3.

The coincidence probabilities as a function of $\theta$ for the hologram with $m=1$ are plotted as red squares in Fig. 3 . For this plot, the distance of the dislocation to the optical axis is set to $d=0.53$ so that the coincidence probability reaches zero at its minimum. The plotted squares deviate from the sinusoidal curve. This simulation result cannot be explained by a simple model that assumes just two states (modes); the effect of excess modes, or highdimensional entanglement, has a nonnegligible contribution.
Similar plots for $m=2$ and $m=3$ are also shown in Fig. 3 . The results show that the deviation from the sinusoidal curve becomes significant as $m$ increases [39]. In the following, we consider the cause of this deviation.

Figure 4 shows the distributions of the azimuthal modes in measurement modes for $m=1,2,3$. The mode weights are calculated as the summation of $p$ from 0 to 30 , i.e., $\sum_{p=0}^{30}\left|\alpha_{p l}^{m, I}\right|^{2}$.

Figure 4 shows that when an $m$-pronged hologram displaced from the center of the optical axis is used, the detection basis state is not in the superposition of just two modes (like $l=0$ and $l=1$ for $m=1$ ), as is assumed in the

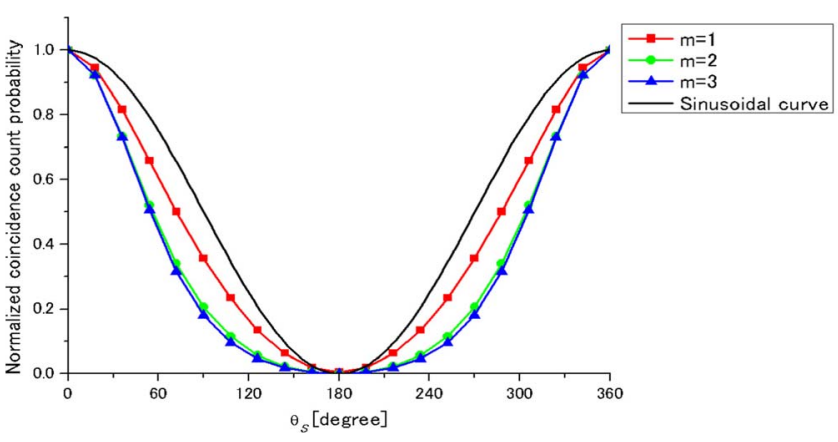

Fig. 3. (Color online) Coincidence fringes when the azimuthal position of $\mathrm{H}_{S}$ is scanned, calculated by using Eq. (8) for $m$ $=1,2,3$ with parameters $w_{P} / w_{0}=2, b / w_{0}=0.102$. The fixed radial positions $d_{S, I} / w_{0}$ are $0.53(m=1), 0.35(m=2)$, and $0.26(m=3)$. The coincidence probability is normalized by the maximum probability in each fringe. 

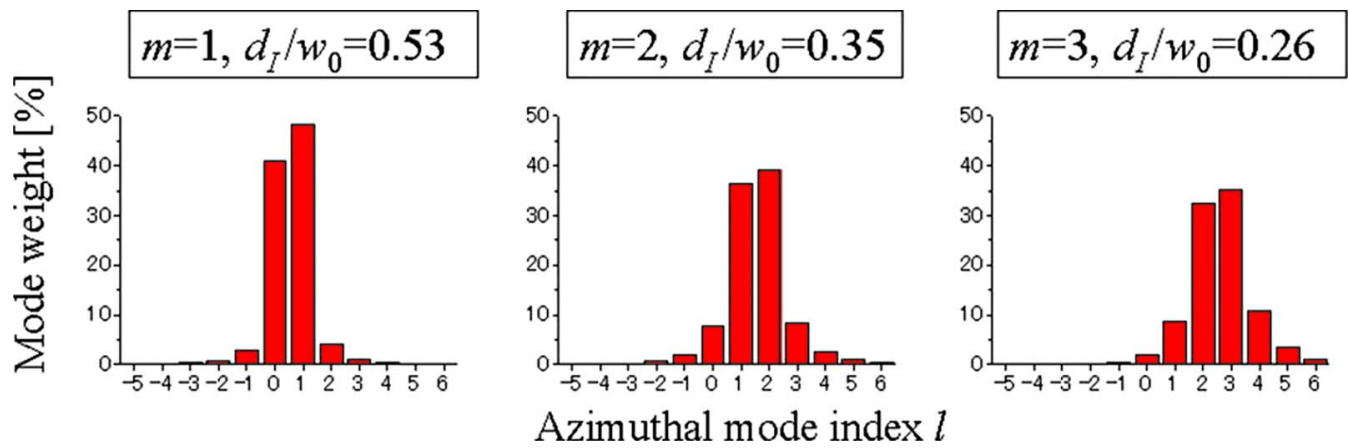

Fig. 4. (Color online) Mode distributions in measurement modes in the idler path for $m=1,2,3$ with $d_{I} / w_{0}=0.53,0.35,0.26$.

simple analysis, but in the superposition of many modes: not only $l=0$ and $l=1$ but also the excess modes ( $l$ $=2,3,4$, and more).

For $m=1$, the portion of the azimuthal modes $l=0$ and $l=1$ to all the modes is $89.3 \%$. In other words, the portion of excess modes is $10.7 \%$. It should also be noted that there are also two dominant azimuthal modes for $m=2$ and $m=3$ : The portion of the azimuthal modes $l=1$ and $l$ $=2$ to all the modes is $75.3 \%$ for $m=2$, and that of $l=2$ and $l=3$ is $68.7 \%$ for $m=3$. However, as $m$ increases to $m=2$ and $m=3$, the portions of excess modes increase to $24.7 \%$ and $31.3 \%$, respectively.

As is discussed below in Subsection 3.C, the emitted photons are in the superposition of many modes from $l=$ $-\infty$ to $l=\infty$. When the mode distribution of the emitted photons is fixed, the deviation from the simple model (sinusoidal curve) should depend on the contribution of the excess modes in the measurement basis state: when the contribution of the excess modes is larger, the deviation is supposed to be larger. As we have seen, the contribution of excess modes in the measurement basis state is larger for larger $m$. Thus, the deviation is expected to be larger for larger $m$. In fact, our numerical result in Fig. 3 meets this expectation.

In summary, the deviation from the sinusoidal curve observed in Fig. 3 is due to the excess modes in the measurement basis states (Fig. 4).

\section{One-Dimensional Scan in the Radial Direction}

Padgett and co-workers suggested $[18,19]$ that quantitative analysis of orbital angular momentum may be possible from the maximum-minimum positions of coincidence counts. They have numerically analyzed the change of the position of the maximum-minimum coincidence probabilities under the condition where the crystal length is negligibly small (thin-crystal limit). In actual experiments, however, crystals with finite length are used, and such finite crystal length may change the mode distribution of the generated entangled state [16] and thus may affect the coincidence count distributions. Here, we investigate in more detail the values and the positions of maximum-minimum coincidence probabilities, based on our present numerical results (Fig. 2) in which the effect of crystal length is taken into account.

The coincidence count probabilities along the $x$ axis $(y$ $=0$ ) in Figs. 2(a)-2(d), which are coincidence probabilities for the hologram with $m=1$, are shown in Fig. 5(a). Note that the calculations take the crystal length $(L=3 \mathrm{~mm}$ for
$\left.\lambda_{P}=351 \mathrm{~nm}\right)$ into account. For comparison, the same coincidence probabilities for a very thin crystal are shown in Fig. 5(b). Calculations for $m=2$ are shown in Fig. 5(c) based on the $2 \mathrm{D}$ data shown in Figs. 2(e)-2(h), which is for the finite crystal length $\left(L=3 \mathrm{~mm}\right.$ for $\left.\lambda_{P}=351 \mathrm{~nm}\right)$, and Fig. 5(d), which is for the case where the crystal length is negligibly small. Note that the plotted data shown in Fig. 5(d) are basically the same as the data shown in Fig. 4(a) in [19].

First, as we discussed in Subsection 3.A, the maximumcoincidence position changes in accordance with the amount of hologram shift in the idler path. However, there are some differences between the cases for finite and zero crystal lengths. The maximum coincidence probability intensities are not uniform for a finite crystal length for different radial positions $d_{I}$ of hologram $I$ [Fig. $5(\mathrm{a})$ ]; the value of the maximum coincidence probability decreases as the hologram dislocation in the idler path approaches the origin. On the other hand, the maximum coincidence probabilities are the same for different $d_{I}$ at the thin-crystal limit [Fig. 5(b)]. This phenomenon becomes more remarkable for $m=2$ [Figs. 5(c) and 5(d)]. The maximum coincidence probabilities are the same for all values of $d_{I}$ when the crystal length is neglected [Fig. 5(d)], while they are not uniform for finite crystal length in Fig. 5(c); the value of the maximum coincidence probability decreases as the hologram dislocation in the idler path approaches the origin, and this tendency becomes more remarkable for $m=2$. More surprisingly, the coincidence count probability is maximum when $d_{S}$ is slightly larger than a given $d_{I}$ [Fig. 5(a)]. Note that for the thincrystal limit the probability is maximum when $d_{S}$ is exactly the same as the given $d_{I}$ [Fig. 5(b)].

We think that the change in the maximum coincidence probability originates from the change of mode distributions for different crystal lengths. As discussed in [16], the mode distribution is given by a Lorentzian-like function with a peak at $l=0$ (Fig. 1 of [16]) for a finite crystal length. The distribution becomes uniform when the crystal length tends to 0 . Thus, for the limit of thin crystal, the probability amplitude of the state $\left|l_{S}=0\right\rangle\left|l_{I}=0\right\rangle$ is the same as that of $\left|l_{S}=-1\right\rangle\left|l_{I}=1\right\rangle$. But for a finite crystal length, the probability amplitude of $\left|l_{S}=0\right\rangle\left|l_{I}=0\right\rangle$ is larger than that of $\left|l_{S}=-1\right\rangle\left|l_{I}=1\right\rangle$. This unbalanced amplitude distribution results in the smaller maximum coincidence probability for $d_{I}=0$, which is proportional to the square of the probability amplitude of $\left|l_{S}=-1\right\rangle\left|l_{I}=1\right\rangle$ component, compared with that for $d_{I}=1.5$, which is given by roughly 

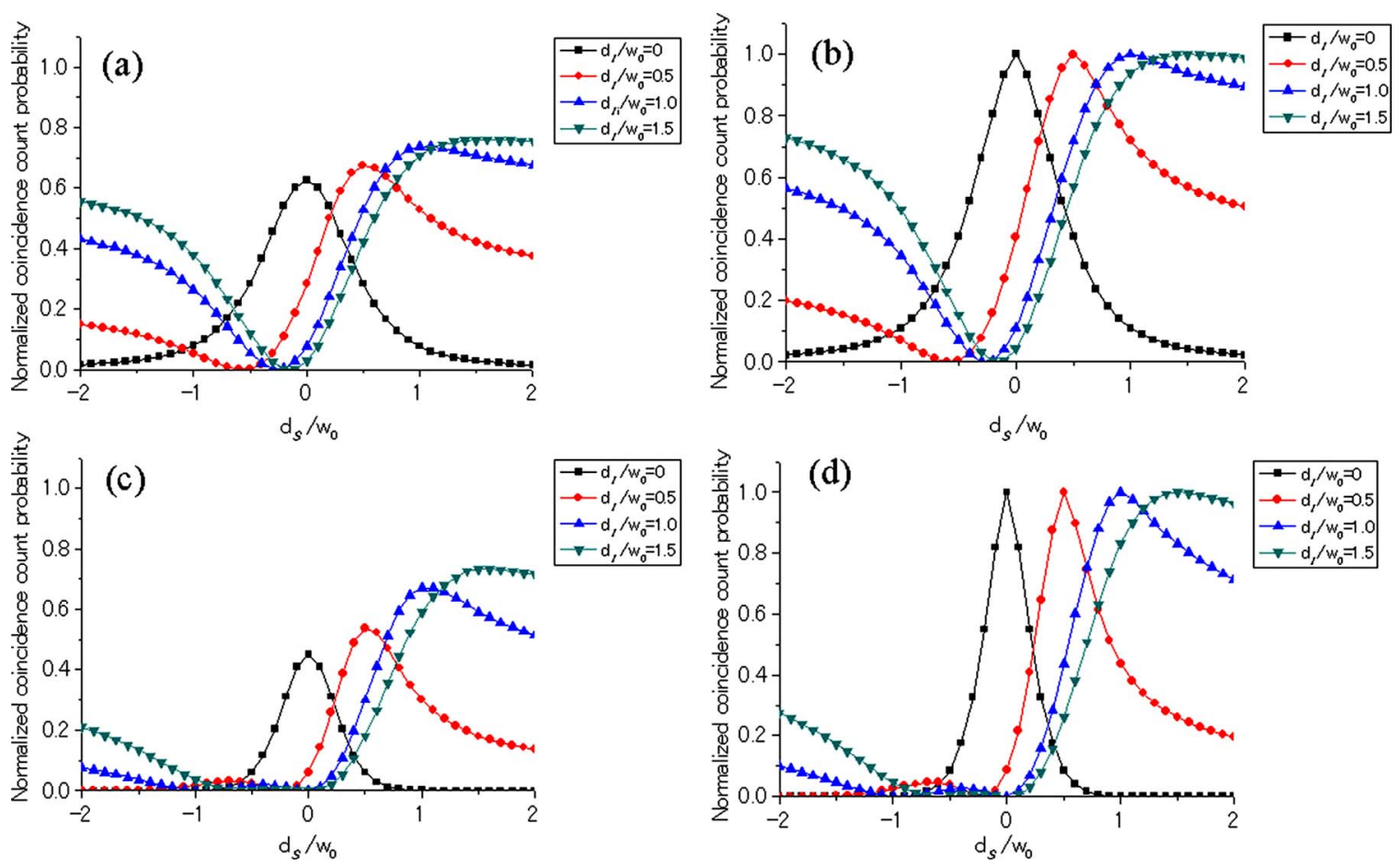

Fig. 5. (Color online) (a), (c) Coincidence probabilities when the radial position of $\mathrm{H}_{S}$ is scanned as calculated by using Eq. (8) for $m$ $=1$ and $m=2$ with parameters $\theta_{I}=\theta_{S}=0, w_{P} / w_{0}=2$, and $b / w_{0}=0.102$. (b), (d) Coincidence probabilities under the assumption that the crystal length $L$ is zero when the radial position of $\mathrm{H}_{S}$ is scanned for $m=1$ and $m=2$ with parameters $\theta_{I}=\theta_{S}=0, w_{P} / w_{0}=2$

the square of the probability amplitude of the $\left|l_{S}=0\right\rangle \mid l_{I}$ $=0\rangle$ component. The change in the maximum coincidence probability becomes more remarkable for $m=2$. This is because the amplitude of $\left|l_{S}=-2\right\rangle\left|l_{I}=2\right\rangle$ is even smaller than that of $\left|l_{S}=-1\right\rangle\left|l_{I}=1\right\rangle$ [Fig. 1 of [13]], and thus the maximum coincidence probability for $d_{I}=0$ for $m=2$ becomes smaller than that for $m=1$.

This unbalance in the probability amplitudes is also why $d_{S}$, which gives the maximum coincidence probability for a given $d_{I}$, is larger than $d_{I}$ [40].

These phenomena should be noted when the maximum-minimum positions of coincidence counts obtained while scanning the hologram positions are used for the quantitative analysis of orbital angular momentum or in the adjustment of the experimental setup in order to observe entanglement in LG modes by using SPDC.

\section{CONCLUSION}

In conclusion, we have analyzed the coincidence count probabilities when the photon pairs entangled in angular momentum are measured by using holograms with an $m$-fold dislocation, considering the nonzero crystal length. We calculated the coincidence probabilities for the cases when the mode-conversion hologram is scanned two dimensionally (the 2D maps). First, we have found that although the positions of maximum-minimum coincidence probabilities are almost the same for different values of $m$, the distribution of coincidence probabilities, especially the area of low coincidence probabilities, strongly depends on $m$. Second, we find that the coincidence probabilities related to azimuthal changes in hologram positions deviate significantly from the sinusoidal curve often assumed in simple analyses. Third, we have found that the crystal length causes the hologram positions to change for the maximum coincidence probabilities, which is due to the change of mode distributions of the generated photon pairs for different crystal lengths. Most of these results originate from the high-dimensional quantum entanglement of the photon pairs generated via the parametric downconversion. An experimental confirmation of our numerical results would be useful to understand the highdimensional entanglement quantitatively, which is currently explored by using multiphoton states alternatively [41].

\section{ACKNOWLEDGMENTS}

The authors thank H. F. Hofmann, K. Tsujino, and R. Okamoto for their helpful discussion. This study was supported in part by Core Research for Evolutional Science and Technology, Japan Science and Technology Agency, Ministry of Internal Affairs and Communication, the Grants-in-Aid from the Japan Science Promotion Society, the Special Coordination Funds for Promoting Science and Technology, and the 21st century Center of Excellence program.

\section{REFERENCES}

1. L. Allen, M. W. Beijersbergen, R. J. C. Spreeuw, and J. P. Woerdman, "Orbital angular momentum of light and the transformation of Laguerre-Gaussian laser modes," Phys. Rev. A 45, 8185-8189 (1992).

2. J. Leach, M. J. Padgett, S. M. Barnett, S. Franke-Arnold, and J. Courtial, "Measuring the orbital angular momentum of a single photon," Phys. Rev. Lett. 88, 257901 (2002). 
3. R. Zambrini and S. M. Barnett, "Quasi-intrinsic angular momentum and the measurement of its spectrum," Phys. Rev. Lett. 96, 113901 (2006).

4. G. F. Calvo, A. Picón, and R. Zambrini, "Measuring the complete transverse spatial mode spectrum of a wave field," Phys. Rev. Lett. 100, 173902 (2008).

5. D. Kaszlikowski, P. Gnacinski, M. Zukowski, W. Miklaszewski, and A. Zeilinger, "Violations of local realism by two entangled $N$-dimensional systems are stronger than for two qubits," Phys. Rev. Lett. 85, 4418-4421 (2000).

6. N. J. Cerf, M. Bourennane, A. Karlsson, and N. Gisin, "Security of quantum key distribution using $d$-level systems," Phys. Rev. Lett. 88, 127902 (2002).

7. H. Nihira and C. R. Stroud, Jr., "Robust multipartite multilevel quantum protocols," Phys. Rev. A 72, 022337 (2005).

8. A. Mair, A. Vaziri, G. Weihs, and A. Zeilinger, "Entanglement of the orbital angular momentum states of photons," Nature 412, 313-316 (2001).

9. A. Vaziri, G. Weihs, and A. Zeilinger, "Experimental twophoton, three-dimensional entanglement for quantum communication," Phys. Rev. Lett. 89, 240401 (2002).

10. N. K. Langford, R. B. Dalton, M. D. Harvey, J. L. O’Brien, G. J. Pryde, A. Gilchrist, S. D. Bartlett, and A. G. White, "Measuring entangled qutrits and their use for quantum bit commitment," Phys. Rev. Lett. 93, 053601 (2004).

11. A. Vaziri, J. W. Pan, T. Jennewein, G. Weihs, and A. Zeilinger, "Concentration of higher dimensional entanglement: qutrits of photon orbital angular momentum," Phys. Rev. Lett. 91, 227902 (2003).

12. J. T. Barreiro, N. K. Langford, N. A. Peters, and P. G. Kwiat, "Generation of hyperentangled photon pairs," Phys. Rev. Lett. 95, 260501 (2005).

13. D. Kawase, Y. Miyamoto, M. Takeda, K. Sasaki, and S. Takeuchi, "Observing quantum correlation of photons in Laguerre Gauss modes using Gouy phase," Phys. Rev. Lett. 101, 050501 (2008).

14. G. Molina-Terriza, A. Vaziri, J. Řeháček, Z. Hradil, and A. Zeilinger, "Triggered qutrits for quantum communication protocols," Phys. Rev. Lett. 92, 167903 (2004).

15. G. Molina-Terriza, J. P. Torres, and L. Torner, "Management of the angular momentum of light: preparation of photons in multidimensional vector states of angular momentum,” Phys. Rev. Lett. 88, 013601 (2002).

16. J. P. Torres, A. Alexandrescu, and L. Torner, "Quantum spiral bandwidth of entangled two-photon states," Phys. Rev. A 68, 050301 (2003).

17. M. Stütz, S. Gröblachera, T. Jennewein, and A. Zeilinger, "How to create and detect $N$-dimensional entangled photons with an active phase hologram," Appl. Phys. Lett. 90, 261114 (2007).

18. S. Franke-Arnold, S. M. Barnett, M. J. Padgett, and L. Allen, "Two-photon entanglement of orbital angular momentum states," Phys. Rev. A 65, 033823 (2002).

19. M. Padgett, J. Courtial, L. Allen, S. Franke-Arnold, S. Barnett, "Entanglement of orbital angular momentum for the signal and idler beams in parametric down-conversion," J. Mod. Opt. 49, 777-785 (2003).

20. A. Ling, A. Lamas-Linares, and C. Kurtsiefer, "Absolute emission rates of spontaneous parametric down-conversion into single transverse Gaussian modes," Phys. Rev. A 77, 043834 (2008).

21. S. S. R. Oemrawsingh, A. Aiello, E. R. Eliel, G. Nienhuis, and J. P. Woerdman, "How to observe high-dimensional two-photon entanglement with only two detectors," Phys. Rev. Lett. 92, 217901 (2004).

22. S. S. Oemrawsingh, X. Ma, D. Voigt, A. Aiello, E. R. Eliel, G. W. 't Hooft, and J. P. Woerdman, "Experimental demonstration of fractional orbital angular momentum entanglement of two photons," Phys. Rev. Lett. 95, 240501 (2005).
23. S. S. Oemrawsingh, J. A. de Jong, X. Ma, A. Aiello, E. R. Eliel, G. W. 't Hooft, and J. P. Woerdman, "Highdimensional mode analyzers for spatial quantum entanglement," Phys. Rev. A 73, 032339 (2006).

24. R. S. Bennink, S. J. Bentley, and R. W. Boyd, "Two-photon' coincidence imaging with a classical source," Phys. Rev. Lett. 9, 113601 (2002).

25. S. Takeuchi, J. Kim, Y. Yamamoto, and H. H. Hogue, "Development of a high-quantum-efficiency single-photon counting system," Appl. Phys. Lett. 74, 1063 (1999).

26. J. Kim, S. Takeuchi, Y. Yamamoto, and H. H. Hogue, "Multi-photon counting using visible light photon counter," Appl. Phys. Lett. 74, 902 (1999).

27. G. Molina-Terriza, S. Minardi, Y. Deyanova, C. I. Osorio, M. Hendrych, and J. P. Torres, "Control of the shape of the spatial mode function of photons generated in noncollinear spontaneous parametric down-conversion," Phys. Rev. A 72, $065802(2005)$

28. A. Valencia, A. Ceré, X. Shi, G. Molina-Terriza, and J. P. Torres, "Shaping the waveform of entangled photons," Phys. Rev. Lett. 99, 243601 (2007).

29. C. H. Monken, P. H. Souto Ribeiro, and S. Pádua, "Transfer of angular spectrum and image formation in spontaneous parametric down-conversion,” Phys. Rev. A 57, 3123-3126 (1998).

30. S. P. Walborn, A. N. de Oliveira, S. Pádua, and C. H. Monken, "Multimode Hong-Ou-Mandel interference," Phys. Rev. Lett. 90, 143601 (2003).

31. For the cases where the Rayleigh range of the pump is smaller than the crystal length, $L$ can be considered the Rayleigh range.

32. G. F. Calvo, A. Picón, and A. Bramon, "Measuring twophoton orbital angular momentum entanglement," Phys. Rev. A 75, 012319 (2007)

33. C. K. Law and J. H. Eberly, "Analysis and interpretation of high transverse entanglement in optical parametric down conversion,” Phys. Rev. Lett. 92, 127903 (2004).

34. M. P. van Exter, A. Aiello, S. S. R. Oemrawsingh, G. Nienhuis, and J. P. Woerdman, "Effect of spatial filtering on the Schmidt decomposition of entangled photons," Phys. Rev. A 74, 012309 (2006).

35. This approximation is introduced to simplify the form of the mode function in position representation, which can be easily calculated numerically.

36. J. P. Torres, Y. Deyanova, L. Torner, and G. Molina-Terriza, "Preparation of engineered two-photon entangled states for multidimensional quantum information," Phys. Rev. A 67, 052313 (2003).

37. These parameters were determined for various experimental conditions.

38. D. Kawase, S. Takeuchi, K. Sasaki, A. Wada, Y. Miyamoto, and M. Takeda, "Determining the optical axes of entangled Laguerre Gauss modes," arXiv.org, arXiv:quant-ph/ $0602199 \mathrm{v} 1$.

39. Note that for $|m|>1$ there are more than one values of the distance $d$ from the dislocation to the optical axis for which the minimum coincidence probability becomes 0 . For the plots in Fig. 3, we selected the smallest values $\left(d / w_{0}\right.$ $=0.35$ for $m=2$ and 0.26 for $m=3$ ) that meet this criterion. We have confirmed that the shape of the plots are almost the same even when we select other values of $d$ for which the minimum coincidence becomes 0 .

40. One can easily confirm this fact by using a simple model assuming the state of the source is given by $|\Psi\rangle=\alpha|0\rangle_{I}|0\rangle_{S}$ $+\beta|1\rangle_{I}|-1\rangle_{S}$ and the detection basis states are $|\psi\rangle_{S}$ $=\sin \delta_{S}|0\rangle_{S}+\cos \delta_{S}|-1\rangle_{S}$ and $|\psi\rangle_{I}=\sin \delta_{I}|0\rangle_{I}+\cos \delta_{I}|1\rangle_{I}$.

41. T. Nagata, R. Okamoto, J. L. O'Brien, K. Sasaki and S. Takeuchi, "Beating the standard quantum limit with four entangled photons," Science 316, 726 (2007). 